Inhalt :

Inhaltsverzeichnis

Neue Namen

Berichtigungen

ZEISSLER, H.: Alexandru V. Grossu - 70 Jahre

Nr. Seiten

CLAUSS, E.: Eine Population von Helicigona (Drobacia) banatica (Roßmaeßler, 1838) in Quedlinburg (Gastropoda, Stylommatophora, Helicidae). $1 \mathrm{Abb}$.

A Population of Helicigona (Drobacia) banatica (Rossmaessler, 1838) at Quedlinburg.

(6) $\quad 85-88$

CLAUSS, E. : Eine albinotische Form von Helix lucorum taurica Krynicki, 1833 (Gastropoda, Stylommatophora, Helicidae). 1 Abb. An Albinotic Form of Helix lucorum taurica Krynicki, 1833.

(8) 105-106

DROZDOWSKI, A.: Ergebnisse bisheriger Untersuchungen über die Schnecken der Wojewodschaften Bydgoszcz, Torun und Włocławek in Polen (Gastropoda). 1 Karte.

Results of the Hitherto Existing Investigations on Gastropods of the Polish Districts Bydgoszcz, Torun, and Włoclawek.

(2) 43-51

DROZDOWSKI, A.: Verzeichnis von Schnecken der polnischen Küstenzone zwischen Gdynia und Gdańsk (Gastropoda). 1 Karte. Species List of Gastropods of the Polish Coastal Region between Gdynia and Gdańsk.

(11) 139-144

DROZDOWSKI, A.: Vergleichende Untersuchungen über Lungen von Eobania vermiculata (Müll.) (Gastropoda, Pulmonata, Helicidae). 3 Abb.

Comparative Investigations on Lungs of Eobania vermiculata (Müll.).

FRANK, CHR.: Ein Beitrag zur Molluskenfauna der Steiermark: Zusammenfassung der Untersuchungen während der Jahre 19651977. 1 Karte.

Contribution to the Fauna of Molluscs in Styria: Survey of Investigations During the Years 1965-1977.

(14) 187-205

FRANK, CHR. : Molluskenfunde im Magen der Maräne Coregonus sp. (Osteichthyes).

Molluscs Found in Stomach Contents of Coregonus sp.

(Osteichthyes).

GROSSU, AL. V.: Revision of the Species of the Daudebardiidae Family of Roumania (Gastropoda, Pulmonata). 19 Figs.

Révision des espèces de la famille de Daudebardiidae de la Roumanie.

GROSSU, AL. V.: Revision der Vitriniden Rumäniens (Gastropoda, Stylommatophora). $8 \mathrm{Abb}$.

Révision des Vitrinides de la Roumanie.

(1) $1-41$

(9) 107-123

GROSSU, AL. V.: Die Variabilität von Bradybaena fruticum (O. F. Müller), mit Beschreibung einer neuen Unterart aus Rumänien (Gastropoda, Stylommatophora, Bradybaenidae). 6 Fig.

La variabilité de Bradybaena fruticum (O. F. Múller) et la description d'une nouvelle sous-espèce en Roumanie.

(23) 303-312 
Nr. Seiten

KNORRE, D. VON: Deroceras sturanyi (Simroth, 1894) im Mittleren Saaletal (Gastropoda, Limacidae). 3 Abb.

Deroceras sturanyi (Simroth, 1894) in the Central Part of the River Saale Valley.

(4) $\quad 75-78$

KÖRNIG, G.: Molluskengesellschaften der Stubnitz (Rügen). Mollusc Associations of Stubnitz (Ruegen).

MATZKE, M.: Landgastropoden innerhalb einer Kleinstadt am Beispiel von Lichtenstein am Fuße des Westerzgebirges.

Terrestrial Gastropods Inside a Small Town at example of Lichtenstein at the Foot of Western Erzgebirge.

$145-167$

MATZKE, M.: Arion fasciatus und Clausilia cruciata am nördlichen Fuß der Bieszczady (VR Polen) (Gastropoda, Stylommatophora). Arion fasciatus and Clausilia cruciata at the Northern Foot of Bieszczady Mountains (Poland).

$301-302$

ZEISSLER, H.: Konchylien aus gelegentlichen Aufschlüssen aus dem Westteil des pleistozänen Travertins von Weimar seit dem Jahre 1964. $6 \mathrm{Abb}$.

Fossil Shelis from Occasional Sections in the Western Part of the Pleistocene Tufa in Weimar since 1964.

ZEISSLER, H.: Konchylien von der Fundstätte des fossilen Elefantenskeletts von Orlishausen in Thüringen. $2 \mathrm{Abb}$.

Shells from the Site of the Fossil Elephant Skeleton of Orlishausen in Thuringia.

ZEISSLER, H.: Mollusken im Waldgebiet zwischen Leipzig-Lauer, Cospuden und Zöbigker (Kr. Leipzig-Land). 1 Karte.

Mollusca in the Forest Area between Leipzig-Lauer, Cospuden and Zöbigker (Distr. Leipzig-Land).

(7) $\quad 89-103$

ZEISSLER, H.: Schnecken in den Restwäldchen zwischen Breitenbich und Dachrieden (Kreis Mühlhausen) sowie aus dem Südteil der Mühlhäuser Hardt (Gastropoda). 1 Karte.

Snails in the Small Forest Parties between Breitenbich and Dachrieden (Distr. Mühlhausen) and in the Southern Part of the Forest "Mühlhäuser Hardt".

(10) $\quad 125-138$

ZEISSLER, H.: Mollusken aus dem Westerwald im Eichsfeld (Westthüringen). 1 Karte.

Mollusca from the Westerwald Mountains in the Eichsfeld (Western Thuringia).

(13) $\quad 169-186$

ZEISSLER, H.: Über die Şchnecken an der Burgruine "Maienluft" bei Wasungen, mit einem Ausblick nach dem Muschelkalkgebiet um Meiningen (Gastropoda).

On the Snails from the Castle Ruin "Maienluft" at Wasungen, with a First Glance at the Triassic Limestone Area round Meiningen (Thuringia).

(15) 207-212

ZEISSLER, H.: Über das Vorkommen von Landschnecken im Bereich des Wartburg-Berges bei Eisenach (Gastropoda).

Land Snails on the Wartburg Mountain near Eisenach.

(17) $\quad 221-228$

ZEISSLER, H.: Neue Bearbeitung der Konchylienfaunen aus dem interglazialen Travertin des Aufschlusses "An der Klippe" in Mühlhausen (Thüringen). $9 \mathrm{Abb}$.

New Research of the Fossil Shells from the Interglacial Calcareous Tufa Exposure "An der Klippe" in Mühlhausen (Thuringia). 
Nr. Seiten

ZEISSLER, H.: Ausführliche Untersuchung der Schneckenfauna im "Eichholz" bei Zwenkau, Kreis Leipzig-Land (Gastropoda). 1 Karte.

Detailed Research on the Snail Fauna in the Forest „Eichholz” near Zwenkau, Distr. Leipzig-Land.

(21) 269-300

Zur B e a c h t ung :

Zu Nr. 19 (ZEISSLER, H., Neubearbeitung Konchylienfaunen „Klippe" Mühlhausen, S. 241-266) befinden sich die Tabellen 1-9 infolge Überformats in einer gesonderten Heftung am Ende dieses Bandes.

Bradybaenidae

\section{Neue Namen}

Bradybaena fruticum popovici-baznosanui subsp. n., p. 310: GROSSU

Daudebardiidae

Daudebardia (Cibinia) cavicola ponorica subsp. n., p. 24 : GROSSU

\section{Berichtigungen}

Zu Nr. 19 (ZEISSLER, H., Neubearbeitung Konchylienfaunen „Klippe“ Mühlhausen, S. 241-266) müssen in den Tabellen 1, 4, 6, 7, 8 folgende Änderungen vorgenommen werden:

Tabelle 1: In der 20. Zeile von unten muß es richtig heißen ", Discus ruderatus".

Tabelle 4: In der 39. Zeile von unten lautet der richtige Prozentwert von Probe 51 "9,81". Tabelle 6: In der 2. Zeile von oben lautet die richtige Anzahl von Belgrandia germanica in Probe 69 "18“.

Tabelle 7: In der 10. Zeile von oben lautet die richtige Schreibweise "Radix peregra aff. obtusa-inflata“.

Tabelle 8: In der 4. Zeile von unten lautet der richtige Prozentwert von Probe 45 „0,12".

\begin{tabular}{llr}
\hline Malak. Abh. Mus. Tierk. Dresden & Band 6 & $1978-1979$ \\
\hline
\end{tabular}

Verantwortlicher Redakteur: Dr. RAINER EMMRICH

Staatliches Museum für Tierkunde Dresden

DDR - 8010 Dresden, Augustusstraße 2

Genehmigt : Ministerrat der Deutschen Demokratischen Republik, Ministerium für Kultur, unter Nr. 105/17/78 u. 105/10/79

Klischees: Graphischer Großbetrieb „Völkerfreundschaft” Dresden Satz und Druck: Graphische Werkstätten Zittau-Görlitz, Betrieb VII Oberlausitzdruck Großschönau III-28-2 